\title{
Efficient fully discrete summation-by-parts schemes for unsteady flow problems: an initial investigation
}

Tomas Lundquist and Jan Nordström

\begin{abstract}
We make an initial investigation into the temporal efficiency of a fully discrete summation-by-parts approach for stiff unsteady flows with boundary layers. As a model problem for the Navier-Stokes equations we consider a two-dimensional advection-diffusion problem with a boundary layer. The problem is discretized in space using finite difference approximations on summation-by-parts form together with weak boundary conditions, leading to optimal stability estimates. For the time integration part we consider various forms of high order summation-by-parts operators, and compare the results to an existing popular fourth order diagonally implicit Runge-Kutta method. To solve the resulting fully discrete equation system, we employ a multi-grid scheme with dual time stepping.
\end{abstract}

\section{Introduction}

Based on finite difference operators on summation-by-parts (SBP) form and the simultaneous-approximation-term (SAT) technique for imposing boundary conditions, the SBP-SAT technique constitutes a robust framework for implementing high order finite difference schemes on complex geometries. By construction, it leads to discrete energy estimates that perfectly imitates the corresponding continuous estimates. This technique was recently extended to initial value problems [2, 3], making it possible to formulate fully discrete SBP-SAT approximations with the same optimal energy estimates. The purpose of this work is to make an initial efficiency study

Tomas Lundquist

Department of Mathematics, Computational Mathematics, Linköping University, SE-581 83 Linköping, Sweden. e-mail: tomas.lunqduist@liu.se

Jan Nordström

Department of Mathematics, Computational Mathematics, Linköping University, SE-581 83 Linköping, Sweden. e-mail: jan.nordstrom@liu.se 
of these new temporal schemes for a stiff model problem with a boundary layer. A more detailed description of this study can be found in [1].

The numerical treatment of unsteady flow problems has gained increased attention in later years due to increased computer resources making realistic calculations of this type more viable. However, the construction of efficient algorithms still remains a significant computational challenge. The two basic methods most commonly used employ Newton iteration and dual time stepping. While Newton iterations are typically better for deep convergence, dual time stepping is more reliable, at least for the initial iterations, and it can also be used for preconditioning purposes [5]. Some studies indicate that a combination of both these techniques can be the most fruitful approach $[4,5,6]$.

To illustrate the SBP-SAT technique for time integration, we consider the test equation $u_{t}+\lambda u=0$ with initial condition $u(0)=f$. The corresponding SBP-SAT approximation of this problem is

$$
D \mathbf{U}+\lambda \mathbf{U}=P^{-1} \sigma\left(U_{0}-f\right) \mathbf{e}_{\mathbf{0}} .
$$

The SAT penalty treatment on the right hand side of (1) forces the solution at $t=0$ to initial data, and the first derivative operator $D$ satisfies the SBP property given by the decomposition $D=P^{-1} Q$, where $Q+Q^{T}=\operatorname{Diag}(-1,0, \ldots, 0,1)$, and $P$ is a positive definite matrix that defines a numerical quadrature. This formulation leads in an automatic way to a clean, optimally sharp energy estimate. With the choice $\sigma=-1$, we get after multiplying (1) with $\mathbf{u}^{*} P$ and adding the conjugate transpose:

$$
\left|u_{N}\right|^{2}+2 \operatorname{Re}(\lambda)|| \mathbf{u}||_{P}^{2}=|f|^{2}-\left|u_{0}-f\right|^{2},
$$

where the norm is defined as $\|\mathbf{u}\|_{P}^{2}=u^{*} P u$. This mimics the continuous energy estimate $|u(T)|^{2}+2 R e(\lambda)\|u\|^{2} \mathrm{~d} t=|f|^{2}$, where $\|u\|^{2}=\int_{0}^{T}|u|^{2} \mathrm{~d} t$ (obtained by multiplying the test equation with $u^{*}$ and then integrating).

As an alternative to the global formulation (1), we may also consider a multistage version with $r+1$ stages:

$$
\begin{aligned}
\left(P^{-1} Q+\lambda I\right) \mathbf{V}^{n+1} & =P^{-1} \sigma\left(V_{0}^{n+1}-U^{n}\right) \mathbf{e}_{\mathbf{0}} \\
U^{n+1} & =V_{r}^{n+1},
\end{aligned}
$$

where $\mathbf{V}^{n+1}=\left(V_{0}^{n+1}, V_{1}^{n+1}, \ldots, V_{r}^{n+1}\right)$. The size of the matrix operator $P^{-1} Q$ in the multi-stage formulation is given by the number of intermediate stages $r+1$ used for each subinterval, and thus remains constant also for long time calculations. Conversely, the minimum number of stages stages depends on how small $P^{-1} Q$ can be made. The classical SBP operators are based on a repeated central finite difference stencil together with boundary closures. An example is the second order operator given by

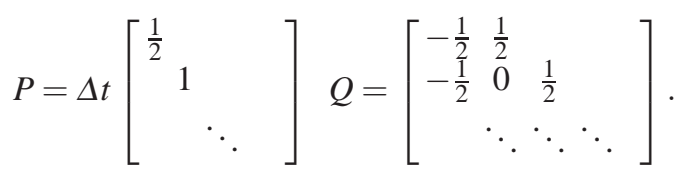


Higher order operators have more extensive boundary closures, thus increasing the minimum number of stages required in the multi-stage approach. Other operators on SBP form, e.g. based on Legendre spectral collocation [9], may alternatively be used to decrease the number of stages necessary, as demonstrated in [12, 13]. See also [10] for more details on the construction non-classical SBP operators based on any type of quadrature.

We summarize the most important advantages of the SBP-SAT technique below.

- The schemes are always A-stable and L-stable. If $P$ is diagonal they are also $B$ stable and preserve energy stability. Moreover, they lead to optimally sharp fully discrete energy estimates.

- The order of convergence is given by the order of accuracy of the quadrature $P$. For classical SBP operators this coincides with the order of the interior scheme.

- The stage order, and thus the order of stiff convergence, is given by the local order of consistency of the operator $P^{-1} Q$. Classical operators are thus limited by the accuracy of the boundary closures.

\section{A stiff flow model in two dimensions}

As a model of the Navier-Stokes equation, we study a viscous fluid undergoing advective flow past a plate with fixed temperature.

$$
\begin{aligned}
u_{t}+u_{x} & =\varepsilon\left(u_{x x}+u_{y y}\right)+\psi & & 0 \leq x, y \leq 1 \quad t \geq 0 \\
u(0, x, y) & =f(x, y) & & t=0 \\
u(t, 0, y)-\varepsilon u_{x}(t, 0, y) & =g_{1}(t, y) & & \partial \Omega_{1}=\{(x, y): x=0\} \\
\varepsilon u(t, 1, y) & =g_{2}(t, y) & & \partial \Omega_{2}=\{(x, y): x=1\} \\
u(t, x, 0) & =0 & & \partial \Omega_{3}=\{(x, y): y=0\} \\
u_{y}(t, x, 1) & =0 & & \partial \Omega_{4}=\{(x, y): y=1\}
\end{aligned}
$$

where $\varepsilon=0.01$. The solid boundary $\partial \Omega_{3}$ is associated with a stiff boundary layer of width $\sqrt{\varepsilon}$, the inflow and outflow boundaries are $\partial \Omega_{1}$ and $\partial \Omega_{2}$ respectively, while $\partial \Omega_{4}$ is a far-field boundary. An exact manufactured solution can be imposed by appropriately specifying the forcing function $\psi$. The energy method yields the estimate

$$
\|u\|_{t}^{2}+2 \varepsilon\left(\left\|u_{x}\right\|^{2}+\left\|u_{y}\right\|^{2}\right)=\int_{\partial \Omega_{1}}\left(g_{1}^{2}-\left(u-g_{1}\right)^{2}\right) \mathrm{d} S+\int_{\partial \Omega_{2}}\left(g_{2}^{2}-\left(u-g_{2}\right)^{2}\right) \mathrm{d} S .
$$

which shows that the problem (2) is well-posed.

In order to resolve the boundary layer around $\partial \Omega_{3}$, we introduce a stretching function $\eta$ of the vertical coordinate, given by

$$
y=1+\frac{\tanh (B(\eta-1))}{\tanh B},
$$


where $B=9 / 4$. This gives $y_{\eta}(0)=\sqrt{\varepsilon}$, and the full stretching function is shown in Figure 1. After this change of coordinate, the model problem (2) becomes

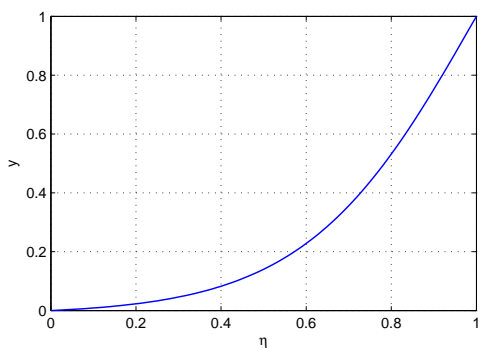

Fig. 1 Stretching of vertical coordinate to resolve the boundary layer.

$$
\begin{aligned}
u_{t}+u_{x} & =\varepsilon\left(u_{x x}+\eta_{y}\left(\eta_{y} u_{\eta}\right)_{\eta}\right)+\psi & & 0 \leq x, \eta \leq 1 \quad t \geq 0 \\
u(0, x, \eta) & =f(x, \eta) & & t=0 \\
u(t, 0, \eta)-\varepsilon u_{x}(t, 0, \eta) & =g_{1}(t, \eta) & & \partial \Omega_{1}=\{(x, \eta): x=0\} \\
\varepsilon u(t, 1, \eta) & =g_{2}(t, \eta) & & \partial \Omega_{2}=\{(x, \eta): x=1\} \\
u(t, x, 0) & =0 & & \partial \Omega_{3}=\{(x, \eta): \eta=0\} \\
\eta_{y} u_{\eta}(t, x, 1) & =0 & & \partial \Omega_{4}=\{(x, \eta): \eta=1\}
\end{aligned}
$$

We now use the techniques outlined in $[7,8]$ to discretize (3) in space using SBPSAT:

$$
\begin{aligned}
U_{t}+\left(P_{x}^{-1} Q_{x} \otimes I_{\eta}\right) U & =\varepsilon\left(\left(\left(P_{x}^{-1} Q_{x}\right)^{2} \otimes I_{\eta}\right) U+\left(I_{x} \otimes\left(H_{y} P_{\eta}^{-1} Q_{\eta}\right)^{2}\right) U\right) \\
& +\left(P_{x}^{-1} \otimes P_{\eta}^{-1} H_{y}\right)\left(\Sigma_{x}(t)+\Sigma_{\eta}(t)\right)+\Psi(t) \\
U(0) & =F
\end{aligned}
$$

where

$$
\begin{aligned}
H_{y} & =\operatorname{Diag}\left(\eta_{y}\right), \quad \Psi(t)=\psi\left(t,\left(\mathbf{x} \otimes \mathbf{1}_{y}\right),\left(\mathbf{1}_{x} \otimes \eta\right)\right) \\
\Sigma_{x}(t) & =\sigma_{0 x}\left(\mathbf{e}_{0 x} \otimes H_{y}^{-1} P_{\eta}\left(\left.\mathbf{u}\right|_{x=0}-\left.\varepsilon \mathbf{u}_{x}\right|_{x=0}-\mathbf{g}_{1}(t)\right)\right) \\
& +\sigma_{1 x}\left(\mathbf{e}_{1 x} \otimes H_{y}^{-1} P_{\eta}\left(\left.\varepsilon \mathbf{u}_{x}\right|_{x=1}-\mathbf{g}_{2}(t)\right)\right) \\
\Sigma_{\eta}(t) & =\sigma_{0 \eta}\left(\left.P_{x} \mathbf{u}\right|_{\eta=0} \otimes \mathbf{e}_{1 \eta}\right)+\sigma_{1 \eta}\left(\left.P_{x} \mathbf{u}_{\eta}\right|_{\eta=1} \otimes \mathbf{e}_{1 \eta}\right) \\
\mathbf{g}_{1}(t) & =g_{1}\left(t,\left(\mathbf{e}_{x 0} \otimes \eta\right)\right), \quad \mathbf{g}_{2}(t)=g_{2}\left(t,\left(\mathbf{e}_{x 1} \otimes \eta\right)\right), \quad F=f\left(\left(\mathbf{x} \otimes \mathbf{1}_{\eta}\right),\left(\mathbf{1}_{x} \otimes \eta\right) .\right.
\end{aligned}
$$

After analyzing (4) using the energy method, we obtain a stable scheme with the following set of penalty parameters: $\sigma_{0 x}=\sigma_{1 x}=-1, \sigma_{1 \eta}=-1 / 2$ and $\sigma_{0 \eta}=$ $-\varepsilon \eta_{y}(0)^{2} /\left(P_{\eta}\right)_{11}$. 


\section{SBP-SAT in time with dual time stepping}

We now consider the semi-discrete problem (4) written in a compact way as

$$
\begin{aligned}
U_{t}+B U & =R(t), 0<t \leq T \\
U(0) & =F .
\end{aligned}
$$

The semi-dicrete spectrum of a fifth order discretization with $N_{x}=N_{\eta}=95$ is shown in Figure 2. The spectral radius of almost $10^{5}$ indicates that an explicit time marching scheme would be an inefficient way to solve this problem. Instead we employ an implicit SBP-SAT time integration scheme with $r+1$ stages:

$$
\begin{aligned}
\left(P^{-1} Q \otimes I_{B}\right) \mathbf{V}^{n+1}+\left(I_{t} \otimes B\right) \mathbf{V}^{n+1} & =\left(P^{-1} \sigma \mathbf{e}_{0}\right) \otimes\left(V_{0}^{n+1}-U^{n}\right)+R \\
U^{n+1} & =V_{r}^{n+1},
\end{aligned}
$$

where $R=\left(R\left(t^{n}\right), R\left(t^{n}+\Delta t / r\right), \ldots, R\left(t^{n}+\Delta t\right)\right)$. Consider the compact form of (5):

$$
\begin{aligned}
\tilde{B} \mathbf{V}^{n+1} & =\tilde{R} \\
U^{n+1} & =V_{r}^{n+1},
\end{aligned}
$$

where $\tilde{B}=P^{-1}\left(Q-\sigma e_{0} e_{0}^{T}\right) \otimes I_{B}+I_{t} \otimes B$. Using the dual time stepping technique, we now employ a multi-grid cycle for solving (6), where the smoothing step consists of stepping forward in pseudo-time toward steady-state. Thus, we add a pseudo time derivative to (6):

$$
\frac{\mathrm{d} \mathbf{V}^{n+1}}{\mathrm{~d} \tau}+\tilde{B} \mathbf{V}^{n+1}=\tilde{R}
$$

To march forward in pseudo-time, we use an explicit $s$-stage low storage RungeKutta smoother:

$$
\begin{aligned}
\mathbf{W}_{0}^{n+1, m+1} & =\mathbf{V}^{n+1, m} \\
\mathbf{W}_{p}^{n+1, m+1} & =\mathbf{V}^{n+1, m}+\Delta \tau \alpha_{p}\left(\tilde{R}-\tilde{B} \mathbf{W}_{p-1}^{n+1, m+1}\right), p=1, \ldots, s \\
\mathbf{V}^{n+1, m+1} & =\mathbf{W}_{s}^{n+1, m+1}
\end{aligned}
$$

The stability function of this scheme is $S(z)=\left(1+\alpha_{s} z\left(1+\alpha_{s-1} z\left(\ldots\left(1+\alpha_{1} z\right) \ldots\right)\right)\right)$. To match the semi-discrete spectrum to the left in Figure 2, we use the 4-stage smoother $\alpha=(0.0178571,0.0568106,0.174513,1)$ proposed in [11]. The stability region of this scheme is shown to the right in Figure 2.

\section{Numerical results}

We employ the manufactured solution $u=\sin (2 \pi(x-t)) e^{\frac{1-y}{\sqrt{\varepsilon}}}$ to (2), and compare the numerical results for a selection of high order temporal schemes. We use both classical diagonal norm operators, denoted $\operatorname{SBP}(2 s, s)$, as well as spectral element 

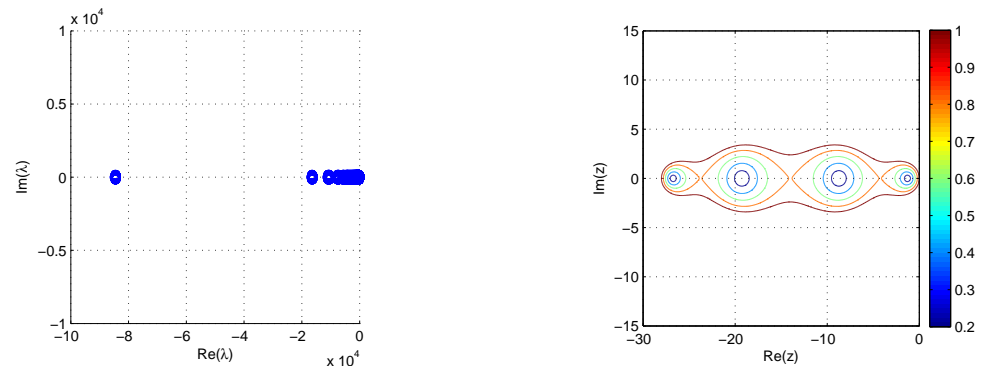

Fig. 2 Left: the semi-discrete spectrum of (4). Right: Stability region of Runge-Kutta smoother.

operators based on Gauss-Lobatto quadrature, denoted by GL( $2 s, s)$. In both cases, $2 s$ denotes the order of the scheme, and $s$ the stage order. For the classical operators we always use the minimum number of stages possible. We use the following operators of order four and eight: $\operatorname{SBP}(4,2)$ with 8 implicit stages, $\operatorname{SBP}(8,4)$ with 16 implicit stages, GL $(4,2)$ with 3 implicit stages, and GL $(8,4)$ with 5 implicit stages. For comparison we also consider a fourth order diagonally implicit Runge-Kutta scheme ESDIRK4, with a stage order of 2, and 5 implicit stages.

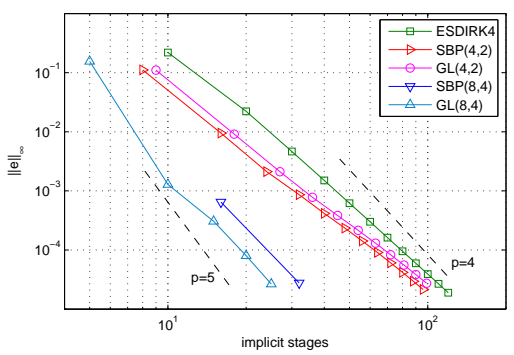

Fig. 3 Accuracy of the high order temporal schemes at $t=1$.

In order to minimize the spatial error component we use the fifth order discretization with $N_{x}=N_{\eta}=95$ with spectrum shown in Figure 2. Note that the spectral radius of almost $10^{5}$ is a result both of the boundary layer and of overresolving in space. The time integration is carried out using a multi-grid V-cycle on three grid levels, with refinement in the vertical coordinate only. On each grid, 10 steps of the explicit Runge-Kutta scheme is used as smoother, with a pseudo-time step restriction of $\operatorname{Re}(z)=-25$ to make the Runge-Kutta scheme stable on each respective grid, see Figure 2. The number of pseudo time iterations is set to make the iteration error less than $10 \%$ compared with the error from the physical time discretization.

In Figure 3 we measure the accuracy at $t=1$ of the different temporal schemes as a function of the total number of implicit stages. In all cases we observe a small level of order reduction, with convergence rates slightly less than the order of the scheme 


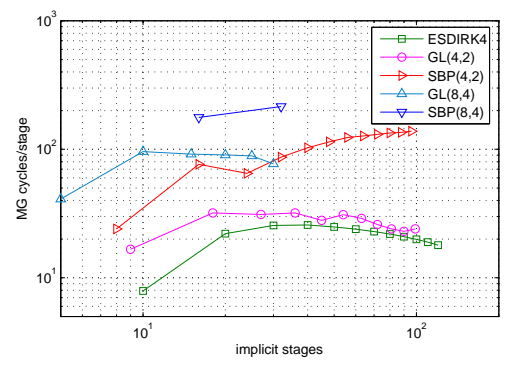

Fig. 4 The amount of work required to resolve each implicit stage.

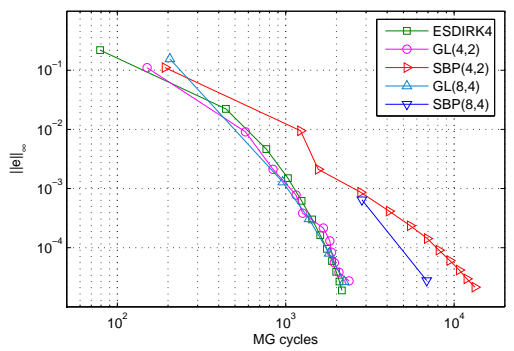

Fig. 5 Accuracy versus the total amount of work required to solve up to $t=1$.

(but higher than the stage order). The number of multi-grid iterations required to converge each implicit stage on average is shown in Figure 4. Figure 5 finally shows the total efficiency, where work is defined as the total number of multi-grid cycles summed over all implicit stages. With this measure the results are comparable between methods using different numbers of implicit stages in each implicit solve. We note that there is no advantage of the diagonally implicit ESDIRK4 method over the Gauss-Lobatto SBP schemes. The classical SBP operators on the other hand, using more implicit stages in each implicit solve, are clearly less efficient here. Current work however indicate that this drawback might be possible to correct by modifying the multi-grid scheme in an appropriate way. Finally, we note that the increased accuracy of the eighth order schemes is counterbalanced by the reduced multi-grid efficiency due to the coarser discretizations in physical time, resulting in very similar results as for the fourth order schemes.

\section{Conclusions and further work}

We have investigated the temporal efficiency of fully discrete SBP-SAT discretizations for unsteady flow calculations. A stiff linear model problem was considered, 
and a a basic dual time-stepping scheme was employed with no attempt made at optimizing the smoother. The numerical results indicate that some of the SBP-SAT time stepping schemes can compete with ESDIRK4 for efficiency already in this basic setting, even though the classical SBP schemes using a larger number of stages did not perform as well. Current work indicate that this disadvantage can be overcome with a more suitable choice of multi-grid scheme that works more efficiently for fully implicit methods.

Future work will aim at developing more efficient multi-grid schemes, including optimization of the Runga Kutta smoother used for pseudo-time stepping. Nonlinear model problems will also be considered, as well as combining the dual time stepping technique with Newton iteration.

\section{References}

1. T. Lundquist \& J. Nordström, Efficient fully discrete summation-by-parts schemes for unsteady flow problems, LiTH- MAT-R, 2014:18, 2014, Department of Mathematics, Linköping University.

2. Nordström, J., Lundquist, T.: Summation-by-parts in time. J. Comput. Phys. 251, 487-499 (2013)

3. Lundquist, T., Nordström, J.: The SBP-SAT technique for initial value problems. J. Comput. Phys. 270, 86-104 (2014)

4. Bijl, H., Carpenter, M.: Iterative solution techniques for unsteady flow computations using high order time integration schemes. Int. J. Numer. Meth. Fluids 47, 857-862 (2005)

5. Knoll, D., Keyes, D.: Jacobian free Newton-Krylov methods: a survey of approaches and applications. J. Comput. Phys. 193, 357-397 (2004)

6. P. Birken, P., Jameson, A.: On nonlinear preconditioners in newtonkrylov methods for unsteady flows. J. Comput. Phys. 62, 565-573 (2010)

7. Nordström, J., Carpenter, M.: High order finite difference methods, multidimensional linear problems and curvilinear coordinates. J. Comput. Phys. 173, 149-174 (2001)

8. Svärd, M., Nordström, J.: A stable high-order finite difference scheme for the compressible Navier-Stokes equations No-slip wall boundary conditions. J. Comput. Phys. 227, 4805-4824 (2008)

9. Carpenter, M., Gottlieb, D.: Spectral methods on arbitrary grids. J. Comput. Phys. 129, 74-86 (1996)

10. Del Rey Fernandez, D., Boom, P., Zingg, D.: A generalized framework for nodal first derivative summation-by-parts operators . J. Comput. Phys.. 266, 214-239 (2014)

11. Kleb, W. L.: Efficient multi-stage time marching for viscous flows via local preconditioning. AIAA J. 99, 181-194 (1999)

12. Boom, P. and Zingg, D.: Runge-Kutta Characterization of the Generalized Summation-byParts Approach in Time arXiv:1410.0202" (2014)

13. Boom, P. and Zingg, D.: High-Order Implicit Time-Marching Methods Based on Generalized Summation-By-Parts Operators. arXiv:1410.0201 (2014) 